\section{Lung Cancer Imaging}

J.G. Ravenel, Ed.

New York, NY: Springer, 2013, 300 pages, \$229

Lung cancer continues to be the leading cause of cancer death in the United States and in developed countries worldwide. The prevention, diagnosis, and treatment of lung cancer will continue to be of major importance in the coming decades. Appropriate therapy depends on accurate staging to determine those cases amenable to surgery and to define the appropriate role for chemotherapy and radiation therapy. Imaging plays a critical role in staging patients with lung cancer. Data from screening trials have shown that the risk of death from lung cancer can be reduced by approximately $20 \%$ when CT screening is applied to higher-risk patients. The iterative emergence of more probing, sensitive imaging technologies has allowed the biologic behavior of lung cancer to be better understood and therapeutic recommendations to be apportioned accordingly. Interventional radiology now offers radiofrequency ablation of specific lesions as a beneficial addition to the therapeutic armamentarium. Another critical piece of this new lung cancer paradigm is an intensely interactive, collaborative, multidisciplinary team approach.

This book was written by current and former faculties at the Medical University of South Carolina, who actively participate as a multidisciplinary thoracic oncology team. The book provides a guide to the diagnosis, staging, and management of lung cancer that can aid in discussions of the therapeutic plan and help patients understand the next steps and what to expect when they come for treatment. The 15 chapters present the epidemiology and classification of lung cancer, screening protocols, imaging in non-small cell lung cancer, preoperative evaluation for lung cancer resection, staging and imaging of small cell lung cancer, surgical treatment of non-small cell lung cancer, the use of radiotherapy in lung cancer, and imaging after lung cancer treatment. Each chapter is well informed, clinically focused, and user-friendly, with current data sources. Unfortunately, several chest radiographs and CT scans presented in chapters $7,9,13$, and 14 are dark and printed on mat paper. The tables and index are helpful.

This book fulfills its intended purpose and meets the needs of its intended audience. It is an authoritative resource for radiologists, oncologists, thoracic surgeons, and pulmonologists. It also will be helpful to general practitioners and to trainees in imaging and thoracic medicine.

\section{E. Edmund Kim}

University of California at Irvine

101 The City Dr. S.

Orange, $C A 92868$

E-mail: edmundek@uci.edu 